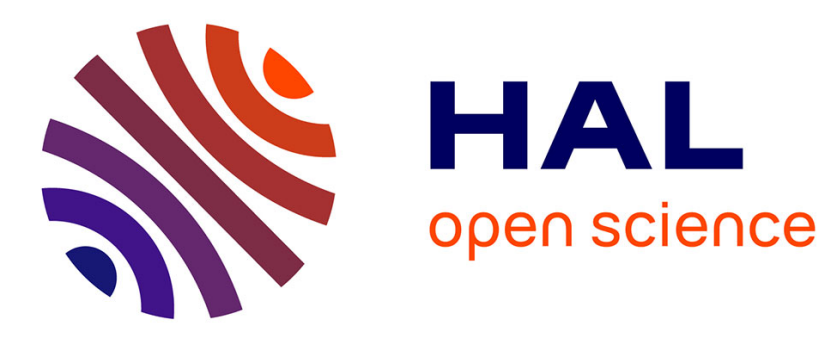

\title{
System F with Coercion Constraints
}

\author{
Julien Cretin, Didier Rémy
}

\section{To cite this version:}

Julien Cretin, Didier Rémy. System F with Coercion Constraints. CSL-LICS 2014: Joint Meeting of the Annual Conference on Computer Science Logic and the Annual Symposium on Logic in Computer Science, Jul 2014, Vienna, Austria. pp.34, 10.1145/2603088.2603128 . hal-01093239

\section{HAL Id: hal-01093239 \\ https://hal.inria.fr/hal-01093239}

Submitted on 10 Dec 2014

HAL is a multi-disciplinary open access archive for the deposit and dissemination of scientific research documents, whether they are published or not. The documents may come from teaching and research institutions in France or abroad, or from public or private research centers.
L'archive ouverte pluridisciplinaire HAL, est destinée au dépôt et à la diffusion de documents scientifiques de niveau recherche, publiés ou non, émanant des établissements d'enseignement et de recherche français ou étrangers, des laboratoires publics ou privés. 


\title{
System F with Coercion Constraints
}

\author{
Julien Cretin Didier Rémy \\ INRIA \\ Julien.Cretin@polytechnique.org Didier.Remy@inria.fr
}

\begin{abstract}
We present a second-order $\lambda$-calculus with coercion constraints that generalizes a previous extension of System $\mathrm{F}$ with parametric coercion abstractions by allowing multiple but simultaneous type and coercion abstractions, as well as recursive coercions and equi-recursive types. This enables a uniform presentation of several type system features that had previously been studied separately: type containment, bounded and instance-bounded polymorphism, which are already encodable with parametric coercion abstraction, and ML-style subtyping constraints. Our framework allows for a clear separation of language constructs with and without computational content. We also distinguish coherent coercions that are fully erasable from potentially incoherent coercions that suspend the evaluation - and enable the encoding of GADTs.

Technically, type coercions that witness subtyping relations between types are replaced by a more expressive notion of typing coercions that witness subsumption relations between typings, e.g. pairs composed of a typing environment and a type. Our calculus is equipped with full reduction that allows reduction under abstractions - but we also introduce a form of weak reduction as reduction cannot proceed under incoherent type abstractions. Type soundness is proved by adapting the step-indexed semantics technique to full reduction, moving indices inside terms so as to control the reduction steps internally - but this is only detailed in the extended version.
\end{abstract}

Categories and Subject Descriptors D.3.1 [Programming Languages]: Formal Definitions and Theory

General Terms Design, Languages, Theory

Keywords Type, Typings, Polymorphism, Coercion, Retyping functions, Type containment, Subtyping, Bounded polymorphism, Type constraints, System F, F-eta, GADTs, Step-Indexed semantics, Full reduction, Recursive coercions, Recursive types

\section{Introduction}

Type systems are syntactical languages to express properties and invariants of programs. Their objects are usually types, typing contexts, and typing derivations. These can be interpreted as mathematical objects or proofs. Typically, a typing judgment $\Gamma \vdash a: \tau$ can be interpreted as a proof that the term $a$ is well-behaved and that

Permission to make digital or hard copies of all or part of this work for personal or classroom use is granted without fee provided that copies are not made or distributed for profit or commercial advantage and that copies bear this notice and the full citation on the first page. Copyrights for components of this work owned by others than ACM must be honored. Abstracting with credit is permitted. To copy otherwise, or republish, to post on servers or to redistribute to lists, requires prior specific permission and/or a fee. Request permissions from permissions@acm.org.

CSL-LICS 2014, July 14-18, 2014, Vienna, Austria.

Copyright (c) 2014 ACM 978-1-4503-2886-9.. \$ \$15.00.

http://dx.doi.org/10.1145/2603088.2603128 its computational behavior is approximated by the type $\tau$ when the approximations of the behaviors of its free variables are given by the typing context $\Gamma$. The simply-typed $\lambda$-calculus extended with constants such as pairs or integers to model the core of a real programming language is the simplest of all type systems. It is also somewhat canonical: it just contains one type construct for each related construct of the language: arrow types $\tau \rightarrow \sigma$ to classify functions, $\tau \times \sigma$ to classify pairs, etc. and nothing else. Each type construct has a counter-part in terms and we may call them computational types.

Simply-typed $\lambda$-calculus is however too restrictive and type systems are usually extended with some form of polymorphism that allows an expression to have several types, or rather a type that stands for a whole collection of other types. Parametric polymorphism, whose System $\mathrm{F}$ is the reference introduces polymorphic types $\forall(\alpha) \tau$. A typing judgment $\Gamma \vdash a: \forall(\alpha) \tau$ means that the program $a$ has also type $\tau[\alpha \leftarrow \sigma]$ (i.e. $\tau$ where $\alpha$ has been replaced by $\sigma$ ) for all types $\sigma$.

This operation, called type instantiation is in fact independent of the program $a$ and can be captured as an auxiliary instantiation judgment $\forall(\alpha) \tau \leq \tau[\alpha \leftarrow \sigma]$. This means that any term that has type $\forall(\alpha) \tau$ also has type $\tau[\alpha \leftarrow \sigma]$. Type instantiation is only a very specific form of some more general concept called type containment introduced by Mitchell (1988) in System F who showed that this is equivalent to closing System $F$ by $\eta$-expansion (hence the name $F_{\eta}$ for the resulting system). Type containment allows instantiation to be performed deeper inside terms (by contrast with System F where it remains superficial), following the structure of types covariantly, or contravariantly on the left of arrow types. Type containment contains the germs of subtyping, which usually refers to a restriction of type containment that does not include type instantiation as part of the subtyping relation, but instead injects primitive subtyping relations between constants such as int $\leq$ float or primitive bottom and top types. The language $\mathrm{F}_{<\text {: }}$ proposed by Cardelli et al. (1994) is the system of reference for combining subtyping with polymorphism. Surprisingly, the languages $F_{\eta}$ and $F_{<:}$share the same underlying concepts but have in fact quite different flavors and are incomparable (no one is strictly more general than the other). For example, $\mathrm{F}_{<:}$has bounded quantification $\forall(\alpha \leq \tau) \sigma$ that allows to abstract over all types $\alpha$ that are a subtype of $\tau$, a concept not present in $\mathrm{F}_{\eta}$. Although quite powerful, bounded quantification seems bridled and somewhat ad hoc as it allows for a unique and upper bound.

The language MLF (Le Botlan and Rémy 2009) is another variant of System $F$ that has been introduced for performing partial type inference while retaining principal types. It has similarities with both $F_{<:}$and $F_{\eta}$, but introduces yet another notion, instance bounded quantification — or unique lower bounds.

In some previous work (Cretin and Rémy 2012), we introduced $\mathrm{F}_{\iota}^{p}$, a language of type coercions with the ability to $a b$ stract over coercions, that can express $\mathrm{F}_{\eta}$ type containment, $\mathrm{F}_{<\text {: }}$ upper bounded polymorphism, and MLF instance-bounded poly- 
morphism, uniformly. Following a general and systematic approach to coercions lead to an expressive and modular design. However, $\mathrm{F}_{\iota}^{p}$ still comes with a severe restriction: abstract coercions must be parametric in either their domain or their codomain, so that abstract coercions are coherent, i.e. their types are always inhabited by concrete coercions. This limitation is disappointing from both theoretical and practical view points. In practice, $F_{L}^{p}$ fails to give an account of subtyping constraints that are used for type inference with subtyping in ML. While in theory, subtyping constraints and second-order polymorphism are orthogonal concepts that should be easy to combine.

Summary of our contributions In this work, we solve this problem and present a language $F_{c c}$ that generalizes $F_{L}^{p}$ (and thus subsumes $F_{\eta}, F_{<:}$, and MLF) to also model subtyping constraints. Besides, $F_{c c}$ includes a general form of recursive coercions, from which we can recover powerful subtyping rules between equirecursive types. As in our previous work, the language is equipped with a full reduction strategy, which also models reduction on open terms and provides stronger properties. We still permit a form of weak reduction on demand to model incoherent abstractions when needed, e.g. to encode GADTs.

We also generalize type coercions to typing coercions which enables a much clearer separation between computational types and erasable types that are all treated as coercions. In particular, type abstraction becomes a coercion and distributivity rules become derivable. Another side contribution of our work which is however not detailed here by lack of space but can be found in the extended version, is an adaption of step-indexed semantics to full reduction strategies, moving indices inside terms.

Plan The rest of the paper is organized as follows. We discuss a few important issues underlying the design of $F_{\mathrm{cc}}$ in $\$ 2$ We present $F_{\mathrm{cc}}$ formally in $\$ 3$ and state its properties in $\$ 4$ We discuss the expressiveness of $F_{c c}$ in $\$ 5$ and differences with our previous work and other related works as well as future works in $\$$

By lack of space, we have omitted many technical details and the whole proof of type soundness via step-indexed terms, which can be found in the extended version of this paper (Cretin and Rémy 2014) and in the first author's PhD dissertation (Cretin 2014 chap 5 ). The language $F_{c c}$ and its soundness and normalization proofs have been formalized and mechanically verified in Coq

\section{The design of $F_{c c}$}

The language $F_{c c}$ is designed around the notion of erasable coercions. Strictly speaking erasable coercions should leave no trace in terms and not change the semantics of the underlying untyped $\lambda$-term. When coercions are explicit and kept during reduction, as in $\mathrm{F}_{\iota}^{p}$, one should show a bisimulation property between the calculus with explicit coercions and terms of $\lambda$-calculus after erasure of all coercions. However, since coercions do not have computational content, they may also be left implicit, as is the case in $F_{c c}$.

Some languages also use coercions with computational content. These are necessarily explicit and cannot be erased at runtime. They are of quite a different nature, so we restrict our study to erasable coercions.

Still, erasability is subtle in the presence of coercion abstraction, because one could easily abstract over nonsensical coercions, e.g. that could coerce any type into any other type. By default, these situations should be detected and rejected of course. We say that a coercion abstraction is coherent when the coercion type is inhabited and incoherent when it may be uninhabited. Notice that type abstraction in System $F$, bounded polymorphism in $F_{<:}$, and instance bounded polymorphism in MLF are all coherent.

\footnotetext{
${ }^{1}$ Scripts are available at http://gallium.inria.fr/ remy/coercions/
}

Coherent abstraction ensures that the body of the abstraction is meaningful-whenever well-typed. Hence, it makes sense to reduce the body of the abstraction before having a concrete value for the coercion- or equivalently to reduce open terms that contain coherent abstract coercions.

Conversely, incoherent abstraction must freeze the evaluation of the body until it is specialized with a concrete coercion that provides inhabitation evidence. Therefore, abstraction over incoherent coercions cannot be erased, even though coercions themselves carry no information and can be represented as the unit type value, as in FC (Weirich et al. 2011) - the internal language of Haskell whose coercion abstractions are (potentially) incoherent.

Choosing a weak evaluation strategy as is eventually done in all programming languages does not solve the problem, but just sweeps it under the carpet: while type-soundness will hold, static type errors will be delayed until applications, and library functions that will never be applicable may still be written.

Conversely, full reduction better exercises the typing rules: that is, type soundness for full reduction provides stronger guarantees. In our view, type systems should be designed to be sound for full reduction even if their reduction is eventually restricted to weak strategies for efficiency reasons. This is how programming languages based on System $F$ or $F_{<:}$have been conceived, indeed.

Therefore, $F_{\mathrm{cc}}$ is equipped with full reduction as the default and this is a key aspect of our design which could otherwise have been much simpler but also less useful.

However, we also permit abstraction over (potentially) incoherent coercions on demand, since this is needed to encode some form of dynamic typing, as can be found in programming languages with GADTs, for example. Indeed, GADTs allow to define parametric functions that are partial and whose body may only make sense for some but not all type instances. When accepting a value of a GADT as argument, the function may gain evidence that some type equality holds and that the value is indeed in the domain of the function. We claim that incoherent abstraction should be used exactly when needed and no more. In particular, one should not make all abstractions incoherent just because some of them must be.

From explicit to implicit coercions Coherence is ensured in $F_{L}^{p}$ by the parametricity restriction that limits abstraction to have a unique upper or lower bound. This also prevents abstract coercions from appearing in between the destructor and the constructor of a redex, a pattern of the form $c\left\langle\lambda\left(x: \tau^{\prime}\right) M\right\rangle N$ (where $c\langle\cdot\rangle$ is the application of a coercion) called a wedge, which could typically block the reduction of explicitly typed terms - therefore loosing the bisimulation with reduction of untyped terms. While the coherence of the abstract coercion $c$ should make it safe to break $c$ apart into two pieces, one attached to the argument $N$, the other one attached to the body $M$, this would require new forms of coercions, new reduction rules, and quite sophisticated typing rules to keep track of the relation between the residual of wedges after they have been split apart. Even though it should be feasible in principle, this approach seemed far too complicated in the general case to be of any practical use.

Therefore our solution is to give up explicit coercions and leave them implicit. While this removes the problem of wedges at once, it also moves us away from a syntactic proof of type soundness. Instead, type soundness in $F_{c c}$ is proved semantically by interpreting types as sets of terms and coercions as proofs of inclusion between types.

Simultaneous coercion abstractions In order to relax the parametricity restriction of $\mathrm{F}_{\iota}^{p}$ and allow coercions whose domain and range are simultaneously structured types, while preserving coherence, we permit multiple type abstractions to be introduced simultaneously with all coercion abstractions that constrain them. 
Since coherence does not come by construction anymore, coherence proofs must be provided explicitly for each block of abstraction as witnesses that the types of coercions are inhabited, i.e. that they can be at least instantiated once in the current environment.

Grouping related abstractions allows to provide coherence proofs independently for every group of abstractions, and simultaneously for every coercion in the same group.

From type coercions to typing coercions A type coercion $\tau \triangleright \sigma$ is a proof that all programs of type $\tau$ have also type $\sigma$ in some environment $\Gamma$. Pushing the idea of coercions further, typings (the pair of an environment and a type, written $\Gamma \vdash \tau$ ) are themselves approximations of program behaviors, which are also naturally ordered. Thus, we may consider syntactical objects, which we call typing coercions, to be interpreted as proofs of inclusions between the interpretation of typings. By analogy with type coercions that witness a subtyping relation between types, typing coercions witness a relation between typings. This idea, which was already translucent in our previous work (Cretin and Rémy 2012), is now internalized.

Interestingly, type generalization can be expressed as a typing coercion-but not as a type coercion: it turns a typing $\Gamma, \alpha \vdash \tau$ into the typing $\Gamma \vdash \forall(\alpha) \tau$. This allows to replace what is usually a term typing rule by a coercion typing rule, with two benefits: superficially, it allows for a clearer separation of term constructs that are about computation from coercion constructs that do not have computational content (type abstraction and instantiation, subtyping, etc.); more importantly, it makes type generalization automatically available anywhere a coercion can be used and, in particular, as parts of bigger coercions. An illustration of this benefit is that the distributivity rules $\left(e . g\right.$. as found in $\left.\mathrm{F}_{\eta}\right)$ are now derivable by composing type generalization, type instantiation, and $\eta$-expansion (generalization of the subtyping rule for computational types).

The advantage of using typing coercions is particularly striking in the fact that all erasable type system features studied in this paper can be expressed as coercions, so that computation and typing features are perfectly separated.

\section{Language definition}

\subsection{The syntax and semantics of terms}

The syntax of the language is given in Figure 1 Because our calculus is implicitly typed, its syntax is in essence that of the $\lambda$-calculus extended with pairs. Terms contain variables $x$, abstractions $\lambda x a$, applications $a b$, pairs $\langle a, b\rangle$, and projections $\pi_{\mathrm{i}} a$ for $i$ in $\{1,2\}$.

Terms also contain two new constructs $\partial a$ and $a \diamond$ called incoherent abstraction and incoherent application, respectively. The incoherent abstraction $\partial a$ can be seen as a marker on the term $a$ that freezes its evaluation, while the incoherent application $a \diamond$ allows evaluation of the frozen term $a$ to be resumed. These two constructs enforce a form of weak reduction in a calculus where full reduction is the default. They are required to model GADTs, but removing them consistently everywhere preserves all the properties of $F_{c c}$; hence, one can always ignore them in a first reading of the paper.

The reduction rules are given on Figure 3 We write $a[x \leftarrow b]$ for the capture avoiding substitution of the term $b$ for the variable $x$ in the term $a$, defined as usual. Head reduction is described by the $\beta$-reduction rule REDAPP, the projection rule REDPROJ, and REDIAPP for unfreezing frozen computations. Reduction can be used under any evaluation context as described by Rule ReDCtx. Evaluation contexts, written $E$, are defined on Figure 2 Since we choose a full reduction relation, all possible contexts are allowed-except reduction under incoherent abstractions: $\partial[]$ is not an evaluation context. Notice that evaluation contexts contain a single node, since the context rule REDCTX can be applied recursively.

$$
\begin{array}{rrr}
\alpha, \beta & & \text { Type variables } \\
x, y & \text { Term variables } \\
a, b::=x|\lambda x a| a a|\langle a, a\rangle| \pi_{\mathrm{i}} a|\partial a| a \diamond & \text { Terms } \\
\kappa::=\star|1| \kappa \times \kappa \mid\{\alpha: \kappa \mid \mathrm{P}\} & \text { Kinds } \\
\tau, \sigma::=\alpha|\tau \rightarrow \tau| \tau \times \tau \mid \forall(\alpha: \kappa) \tau & \text { Types } \\
& |\Pi(\alpha: \kappa) \tau| \mu \alpha \tau|\perp| \top & \\
& |\langle\rangle|\langle\tau, \tau\rangle \mid \pi_{\mathrm{i}} \tau & \text { Coercions } \\
\mathrm{C}::=(\Sigma \vdash \tau) \triangleright \tau & \text { Propositions } \\
\mathrm{P}::=\top|\mathrm{P} \wedge \mathrm{P}|[\mathrm{C}]|\exists \kappa| \forall(\alpha: \kappa) \mathrm{P} & \multicolumn{1}{|c}{\text { Coinduction environments }} \\
\Theta & ::=\varnothing|\Theta, \mathrm{P}| \Theta, \mathrm{P}^{\dagger} \quad \text { Environments } \\
\Gamma::=\varnothing|\Gamma,(\alpha: \kappa)| \Gamma,(x: \tau) & \text { Recursive tokens }
\end{array}
$$

\begin{tabular}{|c|c|}
\hline $\begin{array}{l}\mathrm{REDCTX} \\
\qquad a \leadsto b\end{array}$ & $\begin{aligned}(\lambda x a) b & \leadsto a[x \leftarrow b] \\
\pi_{\mathrm{i}}\left\langle a_{1}, a_{2}\right\rangle & \sim a_{i}\end{aligned}$ \\
\hline$\overline{E[a] \leadsto E[b]}$ & $(\partial a) \diamond \leadsto a$ \\
\hline
\end{tabular}

Figure 1. Syntax

$$
\begin{aligned}
\Sigma & :=\varnothing \mid \Sigma,(\alpha: \kappa) \\
p & ::=x|p v| \pi_{\mathrm{i}} p \mid p \diamond \\
v & :=p|\lambda x v|\langle v, v\rangle \mid \partial a \\
h: & =\lambda x a|\langle a, a\rangle| \partial a \\
D: & =[] a\left|\pi_{\mathrm{i}}[]\right|[] \diamond \\
E: & =\lambda x[]|[] a| a[] \mid[] \diamond \\
& |\langle[], a\rangle|\langle a,[]\rangle \mid \pi_{\mathrm{i}}[]
\end{aligned}
$$

Erasable environments

Prevalues

Values

Constructors

Destructors

Evaluation Contexts

Figure 2. Notations

Figure 3. Reduction relation

The terms we are interested in are the sound ones, i.e. whose evaluation never produces an error. Errors are the subset of syntactically well-formed terms that "we don't want to see" neither in source programs nor during their evaluation: an error is either immediate or occurring in an arbitrary context $E$ (Figure 2); immediate errors are potential redexes $D[h]$ (applications of a destructor $D$ to a constructor $h$ ) that are not valid redexes (the left-hand side of a head-reduction rule). Conversely, values are the irreducible terms that we expect as results of evaluation: they are prevalues, or constructors applied to values-except for incoherent abstractions which may be applied to arbitrary terms; prevalues are variables, or destructors applied to prevalues. Notice that the definition of errors is independent of the reduction strategy while the definition of values is not. This is why we prefer to state soundness as the fact that reduction never produces errors, avoiding the reference to the more fragile definition of values. (In particular, it becomes obvious that a restriction of the reduction strategy may only decrease the reduction relation and therefore the set of errors that can be exposed during the reduction.)

\subsection{Types, kinds, propositions, and coercions}

We use types to approximate the behavior of terms, but types are themselves classified by kinds. So let us present kinds first. Although we do not have type functions, we need to manipulate tuples of types because several type variables and coercion constraints sometimes need to be introduced altogether. For the sake of simplicity and a slight increase in flexibility, we mix types, type sequences, and constrained types into the same syntactical class of 


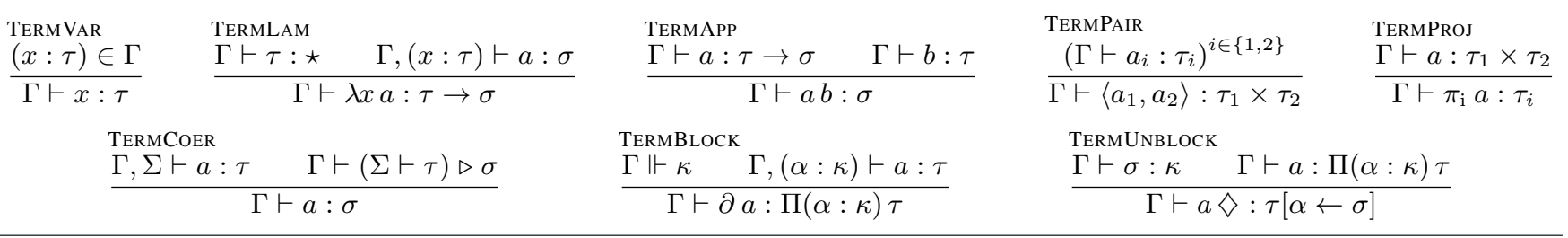

Figure 4. Term typing rules

types which are then classified by kinds. Kinds are written $\kappa$. The star kind $\star$ classifies sets of terms, as usual. The unit kind 1 and the product kind $\kappa \times \kappa$ are used to classify the unit object and pairs of types, which combined together, may encode type sequences: for example, a type variable of kind $\kappa_{1} \times \kappa_{2}$ may stand for a pair of variables of kinds $\kappa_{1}$ and $\kappa_{2}$. The constrained kind $\{\alpha: \kappa \mid \mathrm{P}\}$ restricts the set $\kappa$ to the elements $\alpha$ satisfying the proposition P. For instance, $\{\alpha: \star \mid[\alpha \triangleright \tau]\}$ is the set of types $\sigma$ that can be coerced to (e.g. are a subtype of) $\tau$-assuming that $\alpha$ is not free in $\tau$.

Instead of having only proofs of inclusion between sets of terms, which we call coercions, we define a general notion of propositions, written $\mathrm{P}$. Propositions contain the true proposition $T$, conjunctions $\mathrm{P} \wedge \mathrm{P}$, coercions $[\mathrm{C}]$, coherence propositions $\exists \kappa$, and polymorphic propositions $\forall(\alpha: \kappa) \mathrm{P}$. A coercion $\mathrm{C}$ can only be of the form $(\Sigma \vdash \tau) \triangleright \sigma$. When $\Sigma$ is $\varnothing$, we write $\tau \triangleright \sigma$ for $(\varnothing \vdash \tau) \triangleright \sigma$ and recover the usual notation for type coercions. A coercion can be used directly or seen as a proposition. We write $[\mathrm{C}]$ the use of the coercion $\mathrm{C}$ as a proposition. In a context $\Gamma$, the coercion $(\Sigma \vdash \tau) \triangleright \sigma$ means the existence of a transformation from the typing $\Gamma, \Sigma \vdash \tau$ to the typing $\Gamma \vdash \sigma$. For example, when $\Sigma$ is empty, $\alpha \triangleright \tau$ means that $\alpha$ can be coerced to $\tau$ (e.g. $\alpha$ is a subtype of $\tau$ ).

The coherence proposition of the constrained kind $\{\alpha: \kappa \mid \mathrm{P}\}$, namely $\exists\{\alpha: \kappa \mid \mathrm{P}\}$, gives the usual existential proposition, because coherence corresponds to inhabitation and a type $\tau$ is in the constrained kind $\{\alpha: \kappa \mid \mathrm{P}\}$ if it is in $\kappa$ and satisfies $\mathrm{P}$.

Types are described on Figure 1 They are written $\tau$ or $\sigma$. They contain type variables $\alpha$, arrow types $\tau \rightarrow \sigma$, product types $\tau \times \sigma$, coherent polymorphic types $\forall(\alpha: \kappa) \tau$, incoherent polymorphic types $\Pi(\alpha: \kappa) \tau$, recursive types $\mu \alpha \tau$, the top type $\top$, and the bottom type $\perp$.

Types also contain the unit object \langle\rangle , pairs of types $\langle\tau, \tau\rangle$, and projections $\pi_{\mathrm{i}} \tau$ to construct and project type sequences. We define the projections of pairs $\pi_{\mathrm{i}}\left\langle\tau_{1}, \tau_{2}\right\rangle$ to be equal to the corresponding components $\tau_{i}$. Type equality is then closed by reflexivity, symmetry, transitivity, and congruence for all syntactical constructs. This defines equality judgments on types $\left(\tau_{1}=\tau_{2}\right)$, kinds $\left(\kappa_{1}=\kappa_{2}\right)$, and propositions $\left(\mathrm{P}_{1}=\mathrm{P}_{2}\right)$, which are used in typing rules below. Notice that equality is never applied implicitly.

We use environments to approximate the behavior of variables. The syntax of environments, written $\Gamma$, is described on Figure 1 Environments are lists of type binders $(\alpha: \kappa)$ and term binders $(x$ : $\tau)$. We write $\Sigma$ for environments that do not contain term bindings. We also use lists of propositions, written $\Theta$, called coinduction environments, to keep track of which propositions can be used coinductively.

Let $t$ be a type, a kind, a proposition, a typing environment, a sequence, or a set of such objects. We write $\mathrm{fv}(t)$ the set of free variables of $t$, defined in the obvious way, and $t[\alpha \leftarrow \tau]$ for the capture avoiding substitution of $\tau$ for the variable $\alpha$ in $t$. All objects $t$ are taken up to $\alpha$-conversion of their bound variables.

We assume that environments are well-scoped. That is, $\Gamma,(x$ : $\tau)$ can only be formed when $x \notin \operatorname{dom}(\Gamma)$ and $\operatorname{fv}(\tau) \subseteq \operatorname{dom}(\Gamma)$; and $\Gamma,(\alpha: \kappa)$ can only be formed when $\alpha \notin \operatorname{dom}(\Gamma)$ and $\mathrm{fv}(\kappa) \subseteq \operatorname{dom}(\Gamma)$. Similarly, $\Gamma ; \Theta$ requires $\mathrm{fv}(\Theta) \subseteq \operatorname{dom}(\Gamma)$.

\subsection{Typing judgments}

Types, kinds, and propositions are recursively defined and so are their typing judgments. We actually have the following judgments, all but the term judgment being recursively defined:

$\begin{array}{llll}\Gamma \vdash a: \tau & \text { term } & \Gamma \vdash \Sigma & \text { environment coh. } \\ \Gamma ; \Theta \vdash \mathrm{P} & \text { proposition } & \Gamma \Vdash \Gamma^{\prime} & \text { environment wf } \\ \Gamma ; \Theta \vdash(\Sigma \vdash \tau) \triangleright \sigma & \text { coercion } & \Gamma \Vdash \kappa & \text { kind wf } \\ \Gamma \vdash \tau: \kappa & \text { type } & \Gamma \Vdash \mathrm{P} & \text { proposition wf } \\ \Gamma \vdash \kappa & \text { kind } & \Gamma \Vdash \Theta & \text { coinduction wf }\end{array}$

We assume that judgments are always well-scoped: free variables of objects appearing on the right of the turnstile must be bound in the typing environment $\Gamma$. As usual, the environment $\Gamma$ may not bind (type or term) variable twice, so that it can be considered as a partial mapping from type variables to kinds and term variables to types.

Auxiliary judgments The main judgments are for terms, propositions, and coercions. Others are auxiliary judgments and we describe them first.

The kind judgment $\Gamma \vdash \kappa$ states that the kind $\kappa$ is coherent relatively to the environment $\Gamma$. This judgment is actually equivalent to the proposition judgment $\Gamma \vdash \exists \kappa$ that will be explained below. The environment coherence judgment $\Gamma \vdash \Sigma$ checks that every kind appearing in $\Sigma$ is coherent in the environment that precedes it. It is defined by the two rules:

$$
\Gamma \vdash \varnothing \quad \frac{\Gamma \vdash \Sigma \quad \Gamma, \Sigma \vdash \kappa}{\Gamma \vdash \Sigma,(\alpha: \kappa)}
$$

Kind and proposition well-formedness are recursively scanning their subexpressions for all occurrences of coercion propositions $[(\Sigma \vdash \tau) \triangleright \sigma]$ to ensure that $\Sigma, \tau$, and $\sigma$ are well-typed, as described by the following rule:

$$
\frac{\stackrel{\text { WFPCOER }}{\Gamma \vdash \Sigma} \quad \Gamma, \Sigma \vdash \tau: \star \quad \Gamma \vdash \sigma: \star}{\Gamma \Vdash[(\Sigma \vdash \tau) \triangleright \sigma]}
$$

Well-formedness judgements $\cdot \Vdash \cdot$ recursively check well-formedness of their subcomponents. See the extended version for the complete description of well-formedness rules. We write $\Vdash \Gamma$ for $\varnothing \Vdash \Gamma$.

The type judgment $\Gamma \vdash \tau: \kappa$ is defined in Figure 5 but we only present the most interesting rules. Rule TYPEPACK is used to turn a type $\tau$ of kind $\kappa$ satisfying a proposition $\mathrm{P}$ into a type of the constrained kind $\{\alpha: \kappa \mid \mathrm{P}\}$. Conversely, TYPEUNPACK turns back a type of the constrained kind $\{\alpha: \kappa \mid \mathrm{P}\}$ into one of kind $\kappa$, unconditionally. TYPEMU allows to build the recursive type $\mu \alpha \tau$, which can be formed whenever $\tau$ is productive as stated by the judgment $\alpha \mapsto \tau$ : wf. Other rules are omitted by lack of space.

Extraction property Typing rules are defined so that some invariants hold, such as well-formedness or well-kindedness of subcomponents. Therefore, there are immediate lemmas called extraction lemmas that state these invariants.

- Terms: If $\Gamma \vdash a: \tau$ and $\Vdash \Gamma$ hold, then $\Gamma \vdash \tau: \star$ holds.

- Types: If $\Gamma \vdash \tau: \kappa$ and $\Vdash \Gamma$ hold, then $\Gamma \Vdash \kappa$ holds. 


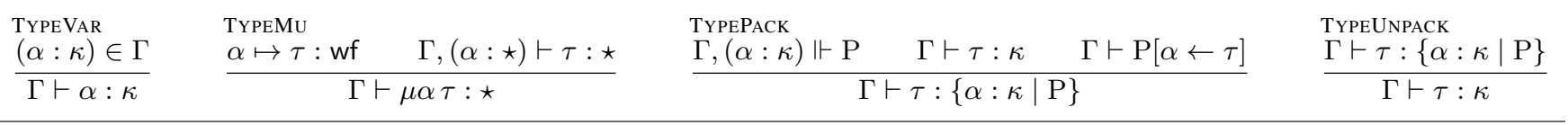

Figure 5. Type judgment relation (excerpt)

- Propositions: If $\Gamma$; $\Theta \vdash \mathrm{P}, \Vdash \Gamma$, and $\Gamma \Vdash \Theta$ hold, then $\Gamma \Vdash \mathrm{P}$ holds.

- Coercions: If $\Gamma ; \Theta \vdash(\Sigma \vdash \tau) \triangleright \sigma$, $\Vdash \Gamma$, and $\Gamma \Vdash \Theta$ hold, then $\Gamma \vdash \Sigma$ holds; moreover if $\Gamma, \Sigma \vdash \tau: \star$ additionally holds, then $\Gamma \vdash \sigma: \star$ holds too.

These invariants also help understanding the typing rules.

Term typing rules Following the tradition, we write $\Gamma \vdash a: \tau$ to mean that in environment $\Gamma$ the term $a$ has type $\tau$. However, we would also like to write this as $a: \Gamma \vdash \tau$ and read it the term a has the typing $\Gamma \vdash \tau$, that is, $a$ is approximated by the type $\tau$ whenever its free variables are in the approximations described by $\Gamma$. We will keep the standard notation to avoid confusion, but we will read the judgment as above when helpful.

Term typing rules are given on Figure 4 Observe that the first five rules are exactly the typing rules of the simply-typed $\lambda$ calculus.

The last two rules are for incoherent abstraction and application (they could be skipped at first): Rule TERMBLOCK says that the program $\partial a$ whose evaluation is frozen may be typed with the incoherent polymorphic type $\Pi(\alpha: \kappa) \tau$ if $a$ can be typed with $\tau$ in an extended context that assigns a well-formed kind $\kappa$ to $\alpha$. Notice that $\Gamma \Vdash \kappa$, as opposed to $\Gamma \vdash \kappa$, does not imply that the kind $\kappa$ is coherent, but only that it is well-formed under $\Gamma$. Rule TermUnBlock is the counterpart of TERMBLOCK. If we have a term $a$ of an incoherent polymorphic type $\Pi(\alpha: \kappa) \tau$, i.e. whose evaluation has been frozen and a type $\sigma$ of kind $\kappa$, we know that the kind $\kappa$ is inhabited by $\sigma$. Therefore, we may safely unfreeze $a$ and give it the type $\tau[\alpha \leftarrow \sigma]$.

Rule TERMCOER is at the heart of our approach which delegates most of the logic of typing to the existence of appropriate typing coercions. The rule reads as follows: if a term $a$ admits the typing $\Gamma, \Sigma \vdash \tau$ and there exists a coercion from $\tau$ to $\sigma$ introducing $\Sigma$ under $\Gamma$, which we write $\Gamma \vdash(\Sigma \vdash \tau) \triangleright \sigma$, then the term $a$ also admits the typing $\Gamma \vdash \sigma$. The presence of $\Sigma$ allows the coercion to manipulate the typing context as well as the type, which is the reason for our generalization from type coercions to typing coercions. When $\Sigma$ is $\varnothing$, the rule looks more familiar and resembles the usual subtyping rule: if a term $a$ has type $\tau$ under $\Gamma$ and there exists a coercion from the type $\tau$ to the type $\sigma$ under $\Gamma$ (which is written $\Gamma \vdash \tau \triangleright \sigma$ ), then the term $a$ has also type $\sigma$ under $\Gamma$.

This factorization of all rules but those of the simply-typed $\lambda$ calculus under one unique rule, namely TERMCOER, emphasizes that coercions are only decorations for terms. Rule TERMCOER annotates the term $a$ to change its typing without changing its computational content, as the resulting term is $a$ itself. This is only made possible by using typing coercions instead of type coercions.

The judgment $\Gamma \vdash(\Sigma \vdash \tau) \triangleright \sigma$ is in fact an abbreviation for the coercion judgment $\Gamma$; $\Theta \vdash(\Sigma \vdash \tau) \triangleright \sigma$ when $\Theta$ is $\varnothing$.

Propositions typing judgment The proposition judgment is defined in Figure 6 As for coercions, we abbreviate $\Gamma ; \Theta \vdash \mathrm{P}$ as $\Gamma \vdash \mathrm{P}$ when $\Theta$ is $\varnothing$. The proposition environment $\Theta$ contains additional hypotheses that can be used coinductively when proving that a coercion holds.

The proposition $[(\Sigma \vdash \tau) \triangleright \sigma]$ is a particular proposition $\mathrm{P}$ stating the existence of a typing coercion from $\tau$ to $\sigma$ introducing $\Sigma$. As described by Rule PropCoer, to be used as a proposition, a coercion must first be typed as such $\Gamma ; \Theta \vdash(\Sigma \vdash \tau) \triangleright \sigma$, which will be explained below, and also satisfy the additional hypothesis $\Gamma, \Sigma \vdash \tau: \star$. This ensures a stronger invariant for propositions than for coercions. As a counterpart, the missing hypothesis is required in (the second clause of) the extaction lemma for the coercion judgment-but it will always be satisfied in the use of coercions in rule TERMCOER, as explained further.

Rule PROPEQ allows the use of type equality. Rule PROPVAR allows the use of a coinductive hypothesis $P$ in $\Theta$. This is written $\mathrm{P}^{\dagger} \in \Theta$ because propositions that are guarded are marked ${ }^{\dagger}$ in $\Theta$ and only those are safe to use coinductively.

In particular, rule PROPFIX which we do not usually find in type systems allows to prove a proposition by coinduction: if $\mathrm{P}$ is true assuming $\mathrm{P}$ in the unguarded coinduction environment, then $\mathrm{P}$ is true without this additional hypothesis. Coinductive propositions are introduced as unguarded so that they cannot be used directly, which would be ill-founded. Only some of the coercion rules (described below) may guard coinduction. The usual rules about recursive types that can be found in other type systems are admissible from the PropFIX rule (see Section 3.3.

Rules Proptrue, PropAndPair, and PropAndProj are uninteresting. Rule PROPFORINTRO and PROPFORELIM are unsurprising. Rule PropExi allows to embed the coherence of a kind $\kappa$, i.e. the existence of a type inhabitant of kind $\kappa$ as the proposition $\exists \kappa$. Rule PROPRES allows to extract a proposition from a type $\tau$ of a constrained kind $\{\alpha: \kappa \mid \mathrm{P}\}$, replacing the variable $\alpha$ of kind $\kappa$ by the witness $\tau$ of kind $\kappa$.

Coercions We now explain typing rules for coercion propositions. We may ignore the environment $\Theta$ in most cases, as it is just unused or transferred to the premises unchanged, except for the three $\eta$-expansion rules that mark the environment as guarded $\Theta^{\dagger}$ in their premises, therefore allowing coinductive uses of propositions $\Theta$ via Rule PropFIX. These are the rules that decompose computational types that have a counterpart in terms, namely CoERARr, COERPROD, and COERPI.

We now explain coercion rules ignoring $\Theta$. Intuitively, the judgment $\Gamma \vdash(\Sigma \vdash \tau) \triangleright \sigma$ implies that any term that admits the typing $\Gamma, \Sigma \vdash \tau$ also admits the typing $\Gamma \vdash \sigma$. (The converse is not true as the coercion typing judgment is semantically incomplete.) One could expect this judgment to be of the form $(\Gamma, \Sigma \vdash \tau) \triangleright(\Gamma \vdash \sigma)$, or even $\left(\Gamma_{1} \vdash \tau_{1}\right) \triangleright\left(\Gamma_{2} \vdash \tau_{2}\right)$. However, in our notation, $\Sigma$ describes environment actions under $\Gamma$ in a compositional manner that eventually permits to go from $\Gamma_{1}$ to $\Gamma_{2}$.

Rule Coerprop is the counter-part of Rule PropCoer, but we do not need additional hypotheses here-since the proposition judgment is stronger than the coercion judgment.

Other coercion typing rules can be understood under the light of Rule TermCoER. In particular, the judgment $\Gamma \vdash(\Sigma \vdash \tau) \triangleright \sigma$ is meant to be used when some judgment $\Gamma, \Sigma \vdash a: \tau$ holds, which by extraction implies that $\Gamma, \Sigma \vdash \tau: \star$ Thus, coercions $\Gamma \vdash(\Sigma \vdash \tau) \triangleright \sigma$ need not assume that $\Gamma, \Sigma \vdash \tau: \star$ holds. However, whenever this is the case, they should then ensure that $\Gamma \vdash \sigma: \star$ holds, as stated by the extraction lemma for coercions.

This explains the difference between Rule COERBот which does not have any premisse and Rule COERTOP, which need one. Otherwise, those two rules are standard and just close the coercion relation with extrema. For any typing $\Gamma \vdash \tau$, there is a smaller typing, namely $\Gamma \vdash \perp$, and a bigger typing, namely $\Gamma \vdash \top$. 


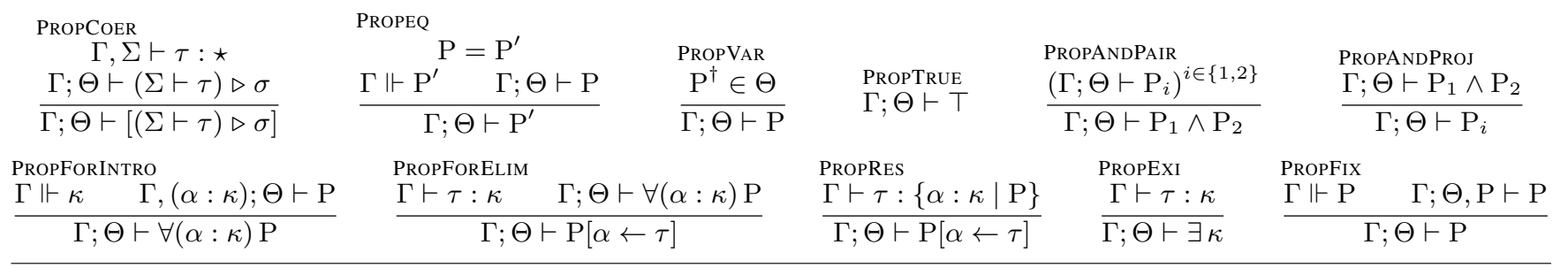

Figure 6. Proposition judgment relation

Rules COERREFl and COERTRAns close the coercion relation by reflexivity and transitivity. To understand COERTRANs let's take a term $a$ with typing $\Gamma, \Sigma_{2}, \Sigma_{1} \vdash \tau_{1}$. Applying Rule TermCoER with the second premise of Rule TERMTRANS ensures that the term $a$ admits the typing $\Gamma, \Sigma_{2} \vdash \tau_{2}$. Applying Rule TERMCOER again with the first premise of Rule TERMTRANS, ensures that $a$ admits the typing $\Gamma \vdash \tau_{3}$ as if we have applied Rule TERMCOER to the original typing of $a$ with the conclusion of Rule COERTRANS.

The Rule CoerWeak implements a form of weakening. It tells that if any term of typing $\Gamma, \Sigma \vdash \tau$ can be seen as $\Gamma \vdash \sigma$, then any term of typing $\Gamma \vdash \tau$ can also be seen as $\Gamma \vdash \sigma$. Since weakening holds for term judgments, we can do the following reasoning to justify this rule. Assuming that the premise $\Gamma, \Sigma \vdash \tau$ holds, we argue that the conclusion should also hold; indeed, a term that admits the typing $\Gamma \vdash \tau$ also admits the typing $\Gamma, \Sigma \vdash \tau$ by weakening; therefore, by the premise of Rule COERWEAK, it must also have typing $\Gamma \vdash \sigma$. However, this reasoning is mathematical and based on our interpretation of coercions: Rule COERWEAK is required as it would not be derivable from other rules-not even admissible - if we removed it from the definition. Notice that this is the only rule that removes binders.

The rules COERProd, COERARr, and COERPI close the coercion relation by $\eta$-expansion, which is the main feature of subtyping. Here, $\eta$-expansion is generalized to typing coercions instead of type coercions. The $\eta$-expansion rules describe how the coercion relation goes under computational type constructors, i.e. those of the simply-typed $\lambda$-calculus.

Intuitively, $\eta$-expansion rules can be understood by decorating the $\eta$-expansion context with coercions at their respective type constructor. These coercions are erasable because the $\eta$-expansion of a term has the same computational behavior as the term itself.

For example, consider the $\eta$-expansion context for the arrow type $\lambda x([] x)$. Placing a term with typing $\Gamma, \Sigma \vdash \tau^{\prime} \rightarrow \sigma^{\prime}$ in the hole, we may give $\lambda x([] x)$ the typing $\Gamma \vdash \tau \rightarrow \sigma$ provided a coercion of type $\Gamma, \Sigma \vdash \tau \triangleright \tau^{\prime}$ is applied around $x$. The result of the application has typing $\Gamma, \Sigma \vdash \sigma^{\prime}$ which can in turn be coerced to $\Gamma \vdash \sigma$ if there exists a coercion of type $\Gamma \vdash\left(\Sigma \vdash \sigma^{\prime}\right) \triangleright \sigma$. Thus, the $\eta$-expansion has typing $\Gamma \vdash \tau \rightarrow \sigma$. While the coercion applied to the result of the application may bind variables $\Sigma$ for the hole (and the argument), the coercion applied to the variable $x$ needs not bind variables, since the variable $x$ could not use them anyway. Notice that the first premise is there to preserve the extraction lemma for coercions.

Rules COERGEN and COERINST implement the main feature of the language, namely simultaneous coherent coercion abstractions. Intuitively, Rule COERGEN combines several type and coercion abstractions. This is however transparent in rule COERGEN since the simultaneous abstractions are grouped in the kind $\kappa$. Hence, this rule looks like a standard generalization rule. The only key here is the premise that requires the kind to be coherent. The coherence hypothesis is necessary for type soundness. Otherwise, it would be possible to type unsound programs by abstracting over some uninhabited kind $\kappa$, such as $\{\alpha: \star \mid[(\alpha \rightarrow \alpha) \triangleright(\alpha \times \alpha)]\}$. Notice that CoerGen is the only rule using typing coercions in a crucial way and that could not be presented as a coercion if we just had type coercions. Rule COERINST is the counter part of COERGEN: it instantiates the abstraction by a type of the right kind.

Rule COERPI is an $\eta$-expansion rule and should be understood by typing the $\eta$-expansion of the incoherent polymorphic type $\partial([] \diamond)$, inserting a coercion around the incoherent application. Placing a term with typing $\Gamma, \Sigma \vdash \Pi\left(\alpha^{\prime}: \kappa^{\prime}\right) \tau^{\prime}$ in the hole, we may first apply weakening to get a typing of the form $\Gamma,(\alpha$ : $\kappa), \Sigma \vdash \Pi\left(\alpha^{\prime}: \kappa^{\prime}\right) \tau^{\prime}$. By instantiation (Rule TERMINST), we get a typing $\Gamma,(\alpha: \kappa), \Sigma \vdash \tau^{\prime}\left[\alpha^{\prime} \leftarrow \sigma^{\prime}\right]$ provided $\Gamma,(\alpha: \kappa), \Sigma \vdash \sigma^{\prime}$ : $\kappa^{\prime}$. Applying a coercion $\left(\Sigma \vdash \tau^{\prime}\left[\alpha^{\prime} \leftarrow \sigma^{\prime}\right]\right) \triangleright \tau$ (Rule TermCoer), we obtain the typing $\Gamma,(\alpha: \kappa) \vdash \tau$, which we may generalize (Rule TERMGEN) to obtain the typing $\Gamma \vdash \Pi(\alpha: \kappa) \tau$ of $\partial([] \diamond)$. Notice that, by contrast with Rule CoERGen, we do not require coherence for the kind $\kappa$, but just its well-formedness. The reason is that this is a term typing rule that blocks the evaluation. However, we require the coherence of the type environment extension $\Sigma$ under $\Gamma$. This is a very important premise because we do not want the incoherence of $\kappa$ to leak in $\Sigma$ and thus under the coercion, because $\eta$-expansions are coercions and thus erasable.

Interestingly, the $\eta$-expansion rules for erasable type constructors are derivable as their introduction and elimination rules are already coercions. For example, consider this simple version of $\eta$ expansion for the universal quantifier:

$$
\frac{\stackrel{\text { CoerAll }}{\Gamma \vdash \kappa} \quad \Gamma,(\alpha: \kappa) ; \Theta \vdash \tau \triangleright \sigma}{\Gamma ; \Theta \vdash \forall(\alpha: \kappa) \tau \triangleright \forall(\alpha: \kappa) \sigma}
$$

Let (1) and (2) be the left and right premises. We have $\Gamma,(\alpha$ : $\kappa) \vdash \alpha: \kappa(3)$ by TyPeVAr. By CoerInst applied to (3) we have $\Gamma,(\alpha: \kappa) ; \Theta \vdash \forall(\alpha: \kappa) \tau \triangleright \tau(\mathbf{4})$. By COERGEN applied to (1) we have $\Gamma ; \Theta \vdash((\alpha: \kappa) \vdash \sigma) \triangleright \forall(\alpha: \kappa) \sigma(\mathbf{5})$. By CoErTrans applied twice to (4), (2), and then (5) we have $\Gamma ; \Theta \vdash((\alpha: \kappa) \vdash$ $\forall(\alpha: \kappa) \tau) \triangleright \forall(\alpha: \kappa) \sigma$, from which we may conclude by Rule COERWEAK.

Similarly, rules for distributing universal types over arrow (COERDISTRIBRIGHT) and product types are also derivable:

$$
\begin{aligned}
& \text { CoerDistribRight } \\
& \Gamma \vdash \kappa \quad \Gamma \vdash \tau: \star \\
& \Gamma ; \Theta \vdash \forall(\alpha: \kappa)(\tau \rightarrow \sigma) \triangleright \tau \rightarrow \forall(\alpha: \kappa) \sigma
\end{aligned}
$$

Rules COERFOLD and COERUNFOLd are the usual folding and unfolding of recursive types, which give the equivalence between $\mu \alpha \tau$ and $\tau[\alpha \leftarrow \mu \alpha \tau]$. Remarkably, the usual rules for reasoning on recursive types (Amadio and Cardelli 1993) are admissible using PROPFIX (we write $\tau_{1} \triangleleft \tau_{2}$ for $\left[\tau_{1} \triangleright \tau_{2}\right] \wedge\left[\tau_{2} \triangleright \tau_{1}\right]$ ):

COERPERIOD

$$
\begin{aligned}
& \alpha \mapsto \sigma: \mathrm{wf} \\
& \frac{\Gamma ; \Theta \vdash\left(\tau_{i} \triangleleft \sigma\left[\alpha \leftarrow \tau_{i}\right]\right)^{i \in\{1,2\}}}{\Gamma ; \Theta \vdash \tau_{1} \triangleright \tau_{2}} \quad \frac{\Gamma,(\alpha, \beta, \alpha \triangleright \beta) ; \Theta \vdash \tau \triangleright \sigma}{\Gamma ; \Theta \vdash \mu \alpha \tau \triangleright \mu \beta \sigma}
\end{aligned}
$$

Interestingly, the proof for COERPERIOD requires reinforcement of the coinduction hypothesis since we need $\tau_{1} \bowtie \tau_{2}$ and not just $\left[\tau_{1} \triangleright \tau_{2}\right]$ in the coinduction hypothesis. 


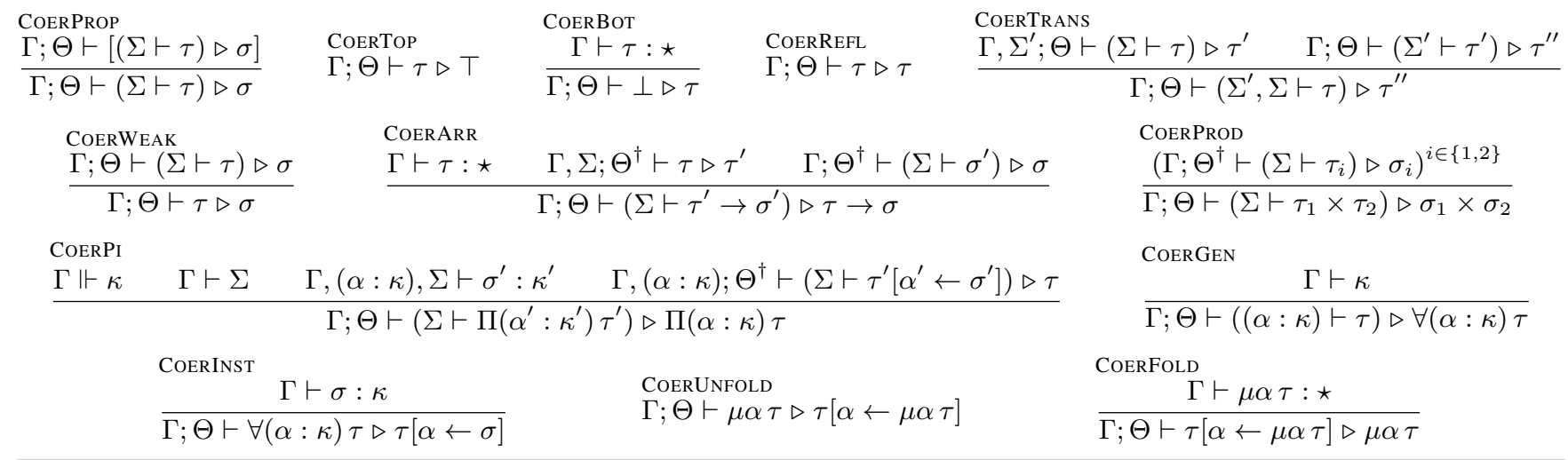

Figure 7. Coercion judgment relation

\begin{tabular}{|c|c|c|c|c|}
\hline \multirow{3}{*}{$\begin{array}{l}\text { RECVAR } \\
\alpha \mapsto \alpha: \text { ne } \\
\quad \text { RECFOR } \\
\quad \alpha \notin \mathrm{fv}(\kappa)\end{array}$} & \multicolumn{2}{|c|}{$\begin{array}{l}\text { RECARR } \\
\left(\alpha \mapsto \tau_{i}: \mathrm{ne}\right)^{i \in\{1,2\}}\end{array}$} & \multicolumn{2}{|c|}{$\begin{array}{l}\text { RECPROD } \\
\left(\alpha \mapsto \tau_{i}: \mathrm{ne}\right)^{i \in\{1,2\}}\end{array}$} \\
\hline & \multicolumn{2}{|c|}{$\alpha \mapsto \tau_{1} \rightarrow \tau_{2}: \mathrm{wf}$} & \multicolumn{2}{|c|}{$\alpha \mapsto \tau_{1} \times \tau_{2}: \mathrm{wf}$} \\
\hline & $\alpha \mapsto \tau: \chi$ & & & $\alpha \mapsto \tau:$ ne \\
\hline \multicolumn{2}{|c|}{$\alpha \mapsto \forall(\beta: \kappa) \tau: \chi$} & \multicolumn{3}{|c|}{$\alpha \mapsto \Pi(\beta: \kappa) \tau: \mathrm{wf}$} \\
\hline $\begin{array}{l}\text { RECMU } \\
\beta \mapsto \tau: \text { wf }\end{array}$ & $\alpha \mapsto \tau: \chi$ & $\begin{aligned} \text { RECW } \\
\alpha \notin\end{aligned}$ & & $\begin{array}{l}\text { RECNE } \\
\alpha \mapsto \tau: \text { wf }\end{array}$ \\
\hline \multicolumn{2}{|c|}{$\alpha \mapsto \mu \beta \tau: \chi$} & $\overline{\alpha \mapsto}$ & & $\overline{\alpha \mapsto \tau: \text { ne }}$ \\
\hline
\end{tabular}

Figure 8. Well-foundedness judgment relation

Finally, the well-foundedness judgment, written $\alpha \mapsto \tau$ : $\chi$, means that $\alpha \mapsto \tau$ is well-founded when $\chi$ is wf or nonexpansive when $\chi$ is ne. The rules are unsurprising. The most interesting ones are RECARR, RECPROD, and RECPI which ensure well-foundedness provided the components are themselves nonexpansive. Conversely, rules RECFOR and RECMU just transfer both well-foundedness and non-expansiveness from their components. For polymorphic types, the abstract variable should not appear in its bound to ensure well-foundedness or non-expansiveness. Of course, recursive types can only be well-formed if they are well founded (left premise of RECMU).

\section{Properties}

System $F_{c c}$ is sound: erroneous terms never appear during the evaluation of well-typed programs. Moreover, in the absence of recursive types and coinduction, all reduction paths terminate. Finally, in the absence of incoherent abstraction, the reduction is confluent.

We loose confluence in the presence of incoherent abstraction, since a partially evaluated term $a$ may be substituted under an incoherent abstraction, after which further reductions won't be permitted inside $a$. This is a well-known problem when mixing full and weak reduction strategies with also a well-known solution (Lévy and Maranget 1999): confluence can easily be restored by using explicit substitutions to hold the substitution at the entry of an incoherent abstraction until the abstraction is applied and evaluation may be resumed. A variant of this solution in the spirit of the work of Blanc et al. (2005) is to add in the language an incoherent weakening construct to cancel the freezing effect of the incoherent abstraction: reduction could still be performed in the weakened part of the incoherent abstraction. This avoids explicit substitutions, but complicates reduction contexts that have to look under incoherent abstractions for occurrences of incoherent weakenings.
Subject reduction is also lost in $\mathrm{F}_{\mathrm{cc}}$-although the language is sound. This is just the consequence of our decision to move from an explicit calculus of coercions to implicit coercions. The type system is just not rich enough to track after reduction the invariants that can be expressed by coercions on source programs, typically when coercions are used in wedges. (This issue is further discussed in $\$ 6.1$ )

The language $F_{c c}$ has been formalized in Coq and its properties have been mechanically verifie ${ }^{2}$

\section{Expressiveness}

The language $\mathrm{F}_{\mathrm{cc}}$ is more expressive than $\mathrm{F}_{l}^{p}$ : apart from the change of presentation, moving from type coercions to typing coercions and from explicit coercions to implicit coercions, the only significant change is for type and coercion abstraction: the new construct of $F_{c c}$ which by design generalizes the two forms of coercion abstraction in $F_{\iota}^{p}$. Indeed, we can choose $\perp$ (resp. T) to witness coercions that are parametric in their domain (resp. range). Therefore the languages $\mathrm{F}_{<:}, \mathrm{MLF}$, and $\mathrm{F}_{\eta}$ which are subsumed by $\mathrm{F}_{\iota}^{p}$ can also be seen as sublanguages of $F_{c c}$.

\subsection{Encoding subtyping constraints}

We claim that languages with ML-like subtyping constraints (Odersky et al. 1999. Pottier 1996) can be simulated in $F_{c c}$. Following the presentation of Pottier (1996), term judgments with subtyping constraints may be written $A \vdash e: \tau \mid C$ where $A$ is the environment, $e$ the expression, $\tau$ its type, and $C$ is a sequence of subtyping constraints.

To ease the embedding of subtyping constraints in $F_{c c}$, we slightly abuse of notations. First, we see let-bindings as the usual syntactic sugar for redexes. We write $\bar{\alpha}$ for a sequence of variables $\alpha_{1} \ldots \alpha_{n}$ where $n$ is left implicit. Given a sequence of variables $\bar{\alpha}$, we see $\alpha_{i}$ as $\alpha . i$, the $i$ 'th projection of $\alpha$. We write $(\bar{\alpha} \mid \mathrm{P})$ as a shorthand for the type binding $\left(\alpha:\left\{\alpha: \star^{n} \mid \mathrm{P}\right\}\right)$ where $n$ is the size of $\bar{\alpha}$. Finally, we see constraint type schemes $\forall \bar{\alpha} . C \Rightarrow \tau$ as the coherent polymorphic type $\forall(\bar{\alpha} \mid C) \tau$.

A term judgment $A \vdash e: \tau \mid C$ can then be seen as the $\mathrm{F}_{\mathrm{cc}}$ judgment $(\bar{\alpha} \mid C), A \vdash e: \tau$ where $\bar{\alpha}$ are free variables of $A, C$, and $\tau$. Notice that the environment in the translation of judgments is always of the form $(\bar{\alpha} \mid C), A$ composed of a single abstraction block $(\bar{\alpha} \mid C)$ followed by term bindings $A$.

Type systems with subtyping constraints use two notions, solvability and consistency, that coincide in ML. Solvability means that one can find a ground instance for type variables that solves the

\footnotetext{
${ }^{2}$ See http://gallium.inria.fr/ $\sim$ remy/coercions/for details.
} 
constraints. Consistency means that the transitive and congruence closure of the set of constraints does not contain inconsistencies.

We claim that solvability of a set of constraints $C$ implies the coherence of the translation of $C$, since it amounts to exhibit type witnesses such that the constraints hold. These witnesses lie in a syntax with simple types and recursive types. Moreover, since solvability is equivalent to consistency, we conclude that consistency is equivalent to coherence.

The two interesting typing judgments for subtyping constraints are for let-binding and subsumption. These are as follows and are admissible in $F_{c c}$ :

$$
\begin{aligned}
& \bar{\alpha} \vdash \bar{\sigma} \quad \bar{\alpha} \vdash C^{\prime}[\bar{\beta} \leftarrow \bar{\sigma}] \\
& \frac{(\bar{\alpha} \mid C), \Gamma,\left(x: \forall\left(\bar{\beta}, C^{\prime}\right) \tau\right) \vdash b: \rho \quad\left(\bar{\alpha}, \bar{\beta} \mid C^{\prime}\right), \Gamma \vdash a: \tau}{(\bar{\alpha} \mid C), \Gamma \vdash(\lambda x b) a: \rho} \\
& \frac{(\bar{\alpha} \mid C), \Gamma \vdash a: \tau \quad\left(\bar{\alpha} \mid C^{\prime}\right) \vdash C \wedge \tau \triangleright \sigma}{\left(\bar{\alpha} \mid C^{\prime}\right), \Gamma \vdash a: \sigma}
\end{aligned}
$$

However, there remain two differences with the way subtyping constraints are usually handled. A judgment $A \vdash e: \tau \mid C$ is valid when $C$ is consistent while our corresponding judgment $(\mathrm{fv}(A, C, \tau) \mid C), A \vdash e: \tau$ is valid when $C$ is solvable, i.e. $\vdash(\mathrm{fv}(C) \mid C)$, which must exhibit a substitution $\theta$ of domain $\mathrm{fv}(C)$ such that $\varnothing \vdash C \theta$. While consistency and solvability coincide in $\mathrm{ML}$ with subtyping constraints, this need not be the case. Consistency is a semantic property while solvability is a syntactic property. Using consistency instead of solvability, we only have to verify a property of the constraints without having to exhibit a concrete solution. Consistency is more flexible than solvability. In practice, it can also be checked more modularly.

We already have some flexibility to reason about coherence in $F_{\mathrm{cc}}$ using propositions and assumptions in the typing context. However, constraint entailment differs in both systems. In particular, we cannot express the decomposition of typing constrains, e.g. deduce the consistency of $\sigma \triangleright \sigma^{\prime}$ from the consistency of $\tau \rightarrow \sigma \triangleright \tau \rightarrow \sigma^{\prime}$, as is the case with subtyping constraints.

The reason is that subtyping constraints are syntactic and taken in a closed-world view: subtyping relations that cannot be expressed syntactically do not hold, which can be used to reinforce constraint entailment. Our approach in $F_{c c}$ is semantic and syntactic coercions must be interpreted in the semantics. Since our semantics has more types and coercions than the syntax allows to build, some reasoning principles that would be true from a purely syntactic point of view will not hold in our semantics and thus cannot be added in the syntax. We are bound to an open-world view. Still, it would be interesting to see how our approach could be extended to allow a form of closed world view and express some negative information.

\subsection{Encoding GADTs}

Incoherent polymorphism is necessary for features that contain some form of dynamic typing, such as GADTs. It may also be a simplification for the programmer that does not have to provide the witness type that proves the coherence-but at his own risk of delaying type errors.

In this subsection we show how GADTs can be encoded with incoherent polymorphism and also how coherence and incoherent polymorphism can be interestingly mixed.

Incoherent polymorphism permits type abstraction for any wellformed kind: inhabited kinds, potentially inhabited kinds, and empty kinds. In the polymorphic type $\Pi(\alpha: \kappa) \tau$, the coherence of kind $\kappa$ may depend on some type variable $\beta$ of the type environment. Depending on how $\beta$ is instantiated, the kind $\kappa$ may or may not be inhabited.
Before we give a concrete example, let us first introduce existential types by their CPS encoding. Because we have two notions of polymorphism, coherent and incoherent, we also have two notions of existential types: we write $\exists(\alpha: \kappa) \tau$ for coherent existential types and $\Sigma(\alpha: \kappa) \tau$ for incoherent existential type $s^{3}$ defined as follows.

$$
\text { coherent: } \quad \exists(\alpha: \kappa) \tau \stackrel{\text { def }}{=} \forall \beta(\forall(\alpha: \kappa)(\tau \rightarrow \beta)) \rightarrow \beta
$$

$$
\text { incoherent: } \quad \Sigma(\alpha: \kappa) \tau \stackrel{\text { def }}{=} \forall \beta(\Pi(\alpha: \kappa)(\tau \rightarrow \beta)) \rightarrow \beta
$$

We define the pack and unpack term syntactic sugar for the coherent existential, and ipack and iunpack for their incoherent version. Notice that the body of the iunpack sugar is hidden under an incoherent type abstraction, and as such is allowed to be unsound because it cannot be reduced.

$$
\begin{aligned}
\text { pack } a \stackrel{\text { def }}{=} \lambda x x a & \text { unpack } a \text { as } x \text { in } b \stackrel{\text { def }}{=} a(\lambda x b) \\
\text { ipack } a \stackrel{\text { def }}{=} \lambda x x \diamond a & \text { iunpack } a \text { as } x \text { in } b \stackrel{\text { def }}{=} a(\partial \lambda x b)
\end{aligned}
$$

Let's assume we have type-level functions and sum types. We can now define the following GADT, named Term, and with kind $\star \rightarrow \star$ (where $\tau \bowtie \sigma$ stands for $[\tau \triangleright \sigma] \wedge[\sigma \triangleright \tau]$ as above):

$$
\begin{aligned}
\text { Term } \alpha & \stackrel{\text { def }}{=} \Sigma\left(\beta: \star \times \star \mid \alpha \bowtie\left(\pi_{1} \beta \rightarrow \pi_{2} \beta\right)\right) \alpha \\
& +\exists \beta \operatorname{Term}(\beta \rightarrow \alpha) \times \operatorname{Term} \beta
\end{aligned}
$$

This GADT is the sum of an incoherent existential type and a coherent one. The incoherent existential type requires $\alpha$ to be an arrow type and stores a term of such type; it also names $\pi_{1} \beta$ the argument type and $\pi_{2} \beta$ its return type. The coherent existential type adds no constraint on $\alpha$ but stores a pair such that its first component applied to its second component is of type $\alpha$; it names $\beta$ the intermediate type. The Term GADT contains two constructors: one for the left-hand side of the sum injecting functions and one for the right-hand side of the sum freezing function applications. We can define its two constructors in the following manner:

$$
\begin{array}{ccl}
\operatorname{Lam} x & \stackrel{\text { def }}{=} & \operatorname{inl}(\text { ipack } x) \\
& : & \forall \alpha \forall \beta(\alpha \rightarrow \beta) \rightarrow \operatorname{Term}(\alpha \rightarrow \beta) \\
\operatorname{App} y x & \stackrel{\text { def }}{=} & \operatorname{inr}(\text { pack }\langle y, x\rangle) \\
& : & \forall \alpha \forall \beta \operatorname{Term}(\alpha \rightarrow \beta) \rightarrow \operatorname{Term} \alpha \rightarrow \operatorname{Term} \beta
\end{array}
$$

We can now define a recursive eval function taking a term of type Term $\alpha$ and returning a term of type $\alpha$ for all type variable $\alpha$. Said otherwise, eval has type $\forall \alpha(\operatorname{Term} \alpha \rightarrow \alpha)$. When the argument is on the left-hand side of the sum, eval simply unpacks it and returns it. When the argument is on the right-hand side of the sum, eval first unpacks it as a pair and applies the evaluation of the first component to the evaluation of the second component. We thus use the incoherent version of unpacking on the left-hand side and the coherent version on the right-hand side.

$$
\begin{aligned}
& \text { eval } x=\text { case } x \text { of } \\
& \left\{\text { inl } x_{1} \mapsto \text { iunpack } x_{1} \text { as } y \text { in } y\right. \\
& \left.\mid \text { inr } x_{2} \mapsto \operatorname{unpack} x_{2} \text { as } y \text { in }\left(\text { eval }\left(\pi_{1} y\right)\right)\left(\text { eval }\left(\pi_{2} y\right)\right)\right\}
\end{aligned}
$$

Let's now suppose that we call eval with a term of type Term $(\tau \times$ $\sigma)$. This term is necessarily from the right-hand side of the sum because $\tau \times \sigma$ cannot be equivalent to an arrow type by consistency. However, in the first branch, in the body of the inconsistent unpack, we have access to the proposition $\tau \times \sigma \triangleleft \pi_{1} \beta \rightarrow \pi_{2} \beta$ which is inconsistent. This sort of inconsistency in some branches of case expressions is frequent with GADTs. Notice however, that we can reduce the second branch because we used a coherent existential type since there is a witness for $\beta$ for any instantiation of $\alpha$.

\footnotetext{
${ }^{3} \Sigma$ here is a binder and has of course no connection with $\Sigma$ used as typing environments.
} 


\section{Discussion}

We first compare $F_{\mathrm{cc}}$ with our prior work and other related works; we then discuss language extensions and future works.

Our version of $F_{c c}$ slightly differs from the original presentation (Cretin 2014) that has been mechanically formalized, but these are purely notational differences, such as merging type and term bindings into a single typing environment.

\subsection{Comparison with $F_{\llcorner}^{p}$}

The closest work is of course our previous work on $F_{L}^{p}$ of which $F_{c c}$ is an extension. The main improvement in $F_{c c}$ is the ability to abstract other arbitrary constrains, but the price to pay is to provide coercion witnesses to ensure the coherence. Notice however, that terms of $F_{\iota}^{p}$ are not affected, since their coherence comes by construction and thus has trivial witnesses.

Coherence is sufficient for type soundness, but in an explicit language of coercions it does not suffice for subject reduction, which also requires that the language has a rich syntactic representation to keep track during the reduction of invariants expressed by coercions. Our approach in $\mathrm{F}_{\mathrm{cc}}$ is to avoid the need for decomposing abstract coercions into smaller ones by presenting an implicit version of the language. This also avoids introducing new coercion constructs in the language and their associated typing rules-which we failed to prove to be sound by syntactic means in an explicit language of coercions.

Therefore, moving from $\mathrm{F}_{\iota}^{p}$ to $\mathrm{F}_{\mathrm{cc}}$ has a cost - the lost of an explicit calculus of coercions with subject reduction. Of course, one can still introduce explicit syntax for coercion typing rules in the source so as to ease type checking, but terms with explicit coercions will not have reduction rules in $\mathrm{F}_{\mathrm{cc}}$.

An interesting question is whether there are interesting languages between $\mathrm{F}_{\iota}^{p}$ and $\mathrm{F}_{\mathrm{cc}}$ that would still have a (relatively simple) calculus of explicit coercions. If we restrict to certain forms of coercions, instead of general coercions, the question of coherence may be much simpler. For example, one could just consider equality coercions as in the language $\mathrm{FC}$ (Weirich et al. 2011).

In $F_{c c}$, we simultaneously abstract over a group of type variables and coercions that constrain those variables. The choice of grouping must be such that the group is coherent for all possible instantiations of variables in the context.

We have also explored a syntactically more atomic version of $F_{c c}$ where type and coercion abstraction are separate constructs as in $\mathrm{F}_{\iota}$ (Cretin and Rémy 2012). Namely, the usual type abstraction $\forall(\alpha) \tau$ and coercion abstraction $\left(\tau_{1} \triangleright \tau_{2}\right) \Rightarrow \sigma$-a term of type $\sigma$ under the hypothesis that $\tau_{1} \triangleright \tau_{2}$ holds. For instance, this would permit to write a function of type $\forall(\alpha)\left(\alpha \rightarrow\left(\tau_{1} \triangleright \tau_{2}\right) \Rightarrow \sigma\right)$ and apply it to a type parameter, then to a value of type $\alpha$, and finally to a coercion. However, this additional flexibility is negligible, these are just $\eta$-expansion variants of terms in $\mathrm{F}_{\mathrm{cc}}$. Moreover, related type abstractions and coercions should still be checked simultaneously. That is, even if the arguments are passed separately, the typing derivation must maintain a notion of grouping underneath so as to check for coherence. The solution in $\mathrm{F}_{\mathrm{cc}}$ seems a better compromise between simplicity and expressiveness.

\subsection{Comparison with other works}

To the best of our knowledge there is no previous work considering erasable typing coercions. Although the typing coercion $(\Sigma \vdash$ $\tau) \triangleright \sigma$ may be simulated as the type coercion $(\forall(\Sigma) \tau) \triangleright \sigma$, there remain two important differences: first, this syntactical play cannot explain type and coercion generalization, and therefore, a type generalization typing rule must still be present at the term level to go from $\Gamma, \Sigma \vdash \tau$ to $\Gamma \vdash \forall(\Sigma) \tau$. Second, coercion rules such as transitivity and $\eta$-expansion rules are more natural when presented as typing coercions, since the type transformation and the environment extension of coercions are presented in parallel, while these two tranformations are confused when presented as type coercions.

Some languages such as Coq or Matita enable instantiation or generalization as coercions (the output type is not polymorphic type while the input type is, or reciprocally), but these are non-erasable coercions that drive the meaning of the underlying program (Coen and Tassi 2009).

The use of type coercions to study features of type system is not at all new. Coercions have also been used in the context of subtyping, but without the notion of abstraction over coercions.

The heavy use of coercions in FC (Weirich et al.|2011), the core language of GHC, was one of our initial motivations for studying coercions in a general setting. In FC, only toplevel coercion axioms coming from type families and newtypes are checked for consistency. Local coercion abstractions are not. This is safe because all coercion abstractions in FC freeze the evaluation. This simplifies the meta-theory but at some significant cost, since the evaluation must be delayed to never reduce in a potentially inconsistent context. Our inconsistent coercions largely coincide to-and was inspired by coercions in FC. In return, $F_{c c}$ offers the ability to choose between coherent and incoherent coercion abstractions so that coherent coercions could be expressed as such and thus not freeze the evaluation and still bring more static guarantees to the user. While $F_{c c}$ treats coercions in the general case, $F C$ considers only a very specific case of equality constraints-with additional restrictionsso that $e . g$. coherence of toplevel coercions axioms can be checked automatically.

Coercions have also been used to eliminate function call overhead from datatype constructors (Vanderwaart et al. 2003): the folding and unfolding of datatype definitions are done using erasable coercions, thus with no run-time effect or hidden cost while preserving the semantics.

Recursive coercions have also been used to provide coercion iterators over recursive structures by Crary (2000). However, the motivations are quite different and coercions are only used as a tool to compile bounded quantification away into intersection types.

\subsection{Variations}

Kind coherence and well-formedness Kind coherence is expressed by the judgment $\Gamma \vdash \kappa$ : which ensures that all coercions contained in $\kappa$ as propositions are also coherent, i.e. intuitively, that they are inhabited whenever their typing context is inhabited. This judgment play a key role in our system. In particular, it is essential in rule COERGEN that the kind of the universal variable be coherence to prevent (erasable) type abstraction of an incoherent kind which would allow typing arbitrary programs, hence some erroneous ones.

However, we also require coherence of all typing contexts introduced by coercions as shown by rule WFPCOER $(\S 3.3$. This is convenient, because we can then assume this coherence whenever we need it. However, this is also odd, because coherence is a semantics issue that we should rather not require globally during well-formedness but only as needed in premises of some typing rules. Besides, this forces all but the term judgment (thus including well-formedness judgments) to be recursively defined. An alternative presentation is also being investigated.

A surface language with explicit non-reducible coercions When coercions are left implicit they must be inferred-as well as coercion witnesses, which is obviously undecidable in the general case. (Typechecking in $F_{\eta}$ or in the most expressive variant of $F_{<:}$are already undecidable.) In practice, the user should provide both of them explicitly-or at least provide sufficient information so that they can be inferred. Hence, a surface language would have explicit coercions just for typing purposes - and coercions should be 
dropped after typechecking. Indeed, in the general case, we do not know how to reduce coercions and, in particular, wedges in a type sound manner. In this setting, our soundness result still appliesreduction will not introduce erroneous programs-but it does not imply subject reduction: it may happen that after reduction there is no way to redecorate the residual program with explicit coercions to make it well-typed. This seems the price to pay for the generality offered by our approach.

There is a large degree of freedom in the design of such a surface language for $F_{c c}$ : it is always possible to be fully explicit by introducing term syntax for describing typing derivations in source terms, hence turning type inference into an easy checking process. However, this would not only contain type annotations but also full construction of coercions (information which is typically inferred in languages such as $\mathrm{F}_{<:}$) and of their coherence proofs (information which is usually obtained by construction). Programming at this level of detail would be too cumbersome for the programmer. Therefore, partial type inference techniques should be used so that sufficient type and coercion information can be reconstructed, but new approaches may be needed.

\subsection{Extensions and variations}

Higher-order types We introduced $F_{c c}$ as an extension of System $F$, thus restricting ourselves to second-order polymorphism. We have verified that our approach extends with higher-order types as in $F_{\omega}$.

Intersection types It should also be possible to add intersection types. (Our semantics already has them.). Following the work of Wells and Haack (2002) on branching types, it would then be interesting to have intersection types as branching typings.

Existential types We haven't included primitive existential types in $F_{\mathrm{cc}}$ but instead used their standard CPS encoding into universal types. Adding primitive existential types would also be interesting but not immediate. This is not so surprising as the combination of existential types with full reduction is known to raise difficulties. A solution we have started to investigate is to use a reduction strategy equivalent to full reduction but where only terms starting with a constructor are substituted. This relates to existing calculi with explicit substitutions and generalizes call-by-need calculi to full reduction.

Side effects We have studied a calculus of coercions in an ideal theoretical setting, but we do not foresee any problem in applying this to a real programming language with impure features such as side effects. We are not bound to full reduction, but on the opposite have all the freedom to choose weak reduction strategies for term abstractions. In the presence of side-effects, we would need a form of value-restriction, allowing type and coercion abstractions only on value forms. We do not expect this to raise new problems with coercions-nor do we expect them to disappear!

\section{Conclusion}

Generalizing the notion of type coercions to typing coercions, we have proposed a type system where the distinction between the computation and the typing aspects of terms have been completely separated. Our apporach subsumes many features of existing type systems including subtyping, bounded quantification, instance bounded quantification, and subtyping constraints.

The soundness of our calculus has been proved using the stepindexed semantics technique which we have adapted to work for calculi with full reduction, just for our needs-but it would be worth exploring this further.

As for coercions, several research directions are worth pursing: the treatment of incoherent coercion abstraction to recover con- fluence; variations on coercion constraints allowing closed-world views; or new type system features such as dependent types.

A prerequisite for using $F_{c c}$ in practice is finding a good surface language with explicit coercions annotations and sufficient type inference. Meanwhile, $F_{c c}$ can still be used to explore (and perhaps simplify) languages with explicit type systems as long as their implicitly typed version is a subset of (some easy extension of) $F_{c c}$.

\section{Acknowledgments}

We thank Gabriel Scherer for fruitful discussions on several aspects of this work.

\section{References}

R. Amadio and L. Cardelli. Subtyping recursive types. ACM TRANSACTIONS ON PROGRAMMING LANGUAGES AND SYSTEMS, 15 (4):575-631, 1993. URL http://doi.acm.org/10.1145/155183. 155231

T. Blanc, J.-J. Lévy, and L. Maranget. Sharing in the weak lambda-calculus. In Processes, Terms and Cycles: Steps on the Road to Infinity, volume 3838 of $L N C S$, pages 70-87. 2005. ISBN 978-3-540-30911-6. . URL http://dx.doi.org/10.1007/11601548_7

L. Cardelli, S. Martini, J. C. Mitchell, and A. Scedrov. An extension of system F with subtyping. Information and Computation, 109(1/2):4-56, 1994. URL http://dx.doi.org/10.1006/inco.1994.1013

C. S. Coen and E. Tassi. Nonuniform Coercions via Unification Hints. In T. Hirschowitz, editor, TYPES, volume 53 of EPTCS, pages 16-29, 2009. URL http://dx.doi.org/10.4204/EPTCS.53.2

K. Crary. Typed compilation of inclusive subtyping. In Proceedings of the International Conference on Functional Programming, 2000. URL http://doi.acm.org/10.1145/351240.351247

J. Cretin. Erasable coercions: a unified approach to type systems. $\mathrm{PhD}$ thesis, Université Paris Diderot, Paris 7, 2014.

J. Cretin and D. Rémy. On the power of coercion abstraction. In Proceedings of the annual symposium on Principles Of Programming Languages, 2012. . URL http://doi.acm.org/10.1145/2103656. 2103699

J. Cretin and D. Rémy. System F with Coercion Constraints. Research Report RR-8456, INRIA, Jan. 2014. URL http://hal.inria.fr/ hal-00934408

D. Le Botlan and D. Rémy. Recasting MLF. Information and Computation, 207(6), 2009. URL http://dx.doi.org/10.1016/j.ic.2008.12. 006

J.-J. Lévy and L. Maranget. Explicit substitutions and programming languages. In Foundations of Software Technology and Theoretical Computer Science, volume 1738 of LNCS, pages 181-200. 1999. ISBN 978-3-540-66836-7. . URL http://dx.doi.org/10.1007/ 3-540-46691-6_14

J. C. Mitchell. Polymorphic type inference and containment. Information and Computation, 2/3(76), 1988. URL http://dx.doi.org/10. 1016/0890-5401(88)90009-0

M. Odersky, M. Sulzmann, and M. Wehr. Type inference with constrained types. Theory and Practice of Object Systems, 5(1):35-55, 1999.

F. Pottier. Simplifying subtyping constraints. In Proceedings of the International Conference on Functional Programming, 1996. URL http: //doi.acm.org/10.1145/232627.232642

J. C. Vanderwaart, D. Dreyer, L. Petersen, K. Crary, R. Harper, and P. Cheng. Typed compilation of recursive datatypes. In Workshop on Types in Language Design and Implementation (TLDI), 2003.

S. Weirich, D. Vytiniotis, S. Peyton Jones, and S. Zdancewic. Generative type abstraction and type-level computation. In Proceedings of the 38th annual ACM SIGPLAN-SIGACT symposium on Principles of programming languages, POPL'11, 2011. URL http://doi.acm.org/ $10.1145 / 1926385.1926411$

J. B. Wells and C. Haack. Branching types. In Proc. of the European Symposium On Programming Languages and Systems, ESOP'02, 2002. URL http://dl.acm.org/citation.cfm?id=645396.651968 